\title{
On the relationship between phylogenetic diversity and trait diversity
}

\author{
Caroline M. Tucker, ${ }^{1,2,9}$ T. Jonathan Davies, ${ }^{3,4,5}$ Marc W. Cadotte,${ }^{6,7}$ and William D. Pearse ${ }^{8}$ \\ ${ }^{1}$ Department of Biology, University of North Carolina at Chapel Hill, Coker Hall, CB \#3280 120 South Road, Chapel Hill, North Carolina \\ 27599-3280 USA \\ ${ }^{2}$ Centre d'Ecologie Fonctionnelle et Evolutive, CNRS, 1919, Route de Mende, Montpellier Cedex 534293 France \\ ${ }^{3}$ Department of Biology, McGill University, 1205 Avenue Docteur Penfield, Montreal, Quebec QC H3A 0G4 Canada \\ ${ }^{4}$ African Centre for DNA Barcoding, University of Johannesburg, PO Box 524, Auckland Park, Johannesburg 2006 South Africa \\ ${ }^{5}$ Departments of Botany, Forest \& Conservation Sciences, University of British Columbia, 6270 University Blvd., Vancouver, \\ British Columbia V6T 1 Z4 Canada \\ ${ }^{6}$ Department of Biological Sciences, University of Toronto-Scarborough, 1265 Military Trail, Toronto, Ontario M1C 1 A4 Canada \\ ${ }^{7}$ Ecology and Evolutionary Biology, University of Toronto, 25 Willcocks St, Toronto, Ontario M5S 3 B2 Canada \\ ${ }^{8}$ Department of Biology \& Ecology Center, Utah State University, Logan, Utah 84322 USA
}

Abstract. Niche differences are key to understanding the distribution and structure of biodiversity. To examine niche differences, we must first characterize how species occupy niche space, and two approaches are commonly used in the ecological literature. The first uses species traits to estimate multivariate trait space (so-called functional trait diversity, FD); the second quantifies the amount of time or evolutionary history captured by a group of species (phylogenetic diversity, PD). It is often-but controversially - assumed that these putative measures of niche space are at a minimum correlated and perhaps redundant, since more evolutionary time allows for greater accumulation of trait changes. This theoretical expectation remains surprisingly poorly evaluated, particularly in the context of multivariate measures of trait diversity. We evaluated the relationship between phylogenetic diversity and trait diversity using analytical and simulation-based methods across common models of trait evolution. We show that PD correlates with FD increasingly strongly as more traits are included in the FD measure. Our results indicate that phylogenetic diversity can be a useful surrogate for high-dimensional trait diversity, but we also show that the correlation weakens when the underlying process of trait evolution includes variation in rate and optima.

Key words: Brownian motion; functional diversity; niche occupancy; phylogeny; trait evolution; traits.

\section{INTRODUCTION}

Essential to much of ecological theory and analysis is the niche concept, and particularly the ecological implications of niche differences among species (Grinnell 1924, Elton 1927, Hutchinson 1961, Chesson 2000, Chase and Leibold 2003). However, measuring the niche space occupied by a group of species directly has proven conceptually and methodologically difficult, in part due to the recognition that species' niches vary across many (frequently difficult to measure and identify) dimensions delineating the conditions under which an organism can grow, survive, and reproduce (Hutchinson 1961, Garnier et al. 2016). Two prominent approaches attempt to overcome this difficulty by estimating niche characteristics either using species' traits (McGill et al. 2006), or using the distances associated with species on a phylogenetic tree (Webb et al. 2002). Both approaches can account for the multidimensionality of the niche: trait-based estimates can incorporate multiple trait measurements assumed to be relevant to niche axes (McGill et al. 2006, Violle and Jiang 2009), while phylogenetic distances should reflect the accumulation of many types of evolved differences among species. More specifically, if some traits can be used as proxies for measuring performance (growth, survival, reproduction)

Manuscript received 20 December 2017; revised 26 February 2018; accepted 21 March 2018. Corresponding Editor: Daniel Charles Laughlin.

${ }^{9}$ E-mail: carolinetucker@unc.edu along relevant environmental gradients, then they should provide information about species' niches (McGill et al. 2006, Violle and Jiang 2009, Garnier et al. 2016). Intuitively, if these traits evolve steadily through time, then the evolutionary relationships among species produced by the patterns of branching on a phylogenetic tree should produce comparable estimates of niche space to those obtained using traits. That logic underlies the use of phylogenetic measures to estimate niche space and differences in the absence of appropriate trait data (Kraft et al. 2007).

A wide range of analyses have considered the total evolutionary history in a set of taxa (phylogenetic diversity, PD), as measured by $\mathrm{PD}_{\text {Faith }}$ [the sum of the branch lengths of a phylogenetic tree (Faith 1992)] and various derived pairwise metrics, to understand the spatial distribution (Davies and Buckley 2011, Meynard et al. 2011), structure (Kraft et al. 2007), and function (Cadotte et al. 2008) of communities (Helmus et al. 2010), ecosystems (Flynn et al. 2011, Srivastava et al. 2012), and regional species pools (Kembel and Hubbell 2006), implicitly or explicitly treating PD as a proxy for unmeasured trait diversity. As species in an assemblage are more distantly related and encompass greater spans of independent evolutionary time (i.e., greater PD), they should have the opportunity to accumulate greater variation in the ecological traits that influence where species can live and their interactions with other species (Webb et al. 2002). If the accumulation of such ecological trait diversity corresponds with occupancy of niche space, then it follows that the niche space occupied by a group of species is determined 
by the total amount of PD and, as a corollary, the structure (topology and relative branch lengths) of the phylogenetic tree. However, the assumptions underlying the PD-asproxy-for-trait diversity claim have frequently been challenged based on evolutionary theory (Futuyma 2010, Cadotte et al. 2017), while empirical support is mixed (Silvertown et al. 2006, Ackerly 2009, Cadotte et al. 2009, Devictor et al. 2010, Kelly et al. 2014, Faith 2015b, Gerhold et al. 2015 for a review, Mazel et al. 2017).

Some of the scepticism and confusion about the relationship between PD and trait diversity may be because PD was initially valued as a biodiversity measure for its perceived ability to capture "feature diversity", where "features" represent homologous traits (Faith 1992). Under this definition, a strong relationship between the two measures is expected; however this particular conceptualization is rarely applied outside of the conservation literature (e.g., Faith 2016). Here we define "trait diversity" more broadly to include the variation in all ecological or 'functional' (Violle et al. 2007) traits, which include a wide variety of physiological, phenological, morphological, and behavioural measures, many of which exhibit complex evolutionary trajectories. Such traits can be related to a phylogeny via macroevolutionary models, making this definition more appropriate for tests of niche occupancy related to the accumulation of ecological trait differences among species. Note we will refer to trait diversity as 'FD' after functional diversity, for consistency with the existing literature.

Surprisingly, the theoretical basis for treating PD as a proxy for trait diversity has not received thorough assessment in the ecological literature, relying primarily on verbal arguments (but see Letten and Cornwell 2015, Cadotte et al. 2017). In particular, trait-based approaches to ecology increasingly emphasize multi-dimensional measures providing estimates of species' ecological niches (Villéger et al. 2008, Blonder et al. 2014). Yet we lack a clear understanding of whether calculating FD using multiple traits might influence the shape or strength of the PD-FD relationship, as past analyses have been typically limited to one or two traits (Kraft et al. 2007, Letten and Cornwell 2015). In addition, the tempo and mode of evolution may greatly affect the nature and strength of the relationship (Ackerly 2009, Futuyma 2010, Cadotte et al. 2017) but the ecological literature has primarily focussed on the expectations under Brownian motion (Kraft et al. 2007, Letten and Cornwell 2015). By evaluating the expected relationship between PD and FD under an expanded set of evolutionary models, we can then identify when (or if) PD should be expected to be a useful proxy for FD in ecological analyses.

In this work, we evaluate whether the trait diversity of an assemblage is expected to be positively related to the phylogenetic diversity, and ask whether trait dimensionality, the form of evolutionary model, or evolutionary complexity (number of parameters in the evolutionary models) alters this relationship. We focus on the clade-wide relationship between phylogenetic diversity and trait diversity; although ecologists are often interested in estimating niche differences in local communities (e.g., Helmus et al. 2010) or for choosing sets of species to prioritize for conservation (Mazel et al. 2017), without first clarifying expectations for the PD-FD relationship at the clade scale it is not possible to formulate null expectations against which to interpret observations in local communities. Thus, our results are applicable not just to community ecological studies, but any study that implicitly or explicit relies on a strong correlation between PD and FD. We consider evolutionary models that allow for traits to continuously diverge over time (i.e. Brownian motion or $\mathrm{BM}$ ) and those that constrain trait divergence such as by stabilizing selection (i.e., the Ornstein-Uhlenbeck model or OU). We also provide results for more complex models that allow for variation in the rate and optimum of trait evolution among clades, and those where rates of trait divergence change through time (i.e., Early Burst or EB). In doing so we hope to provide a theoretical background against which to inform the use of PD in future ecological analyses.

\section{Methods}

\section{Measuring phylogenetic and trait diversity}

$\mathrm{PD}_{\text {Faith }}$ is calculated as the total sum of the branch lengths on a tree (Faith 1992). We estimated FD using the functional richness metric, which calculates the volume of the multidimensional convex hull encompassing all ordinated trait values in a set (Cornwell et al. 2006, Villéger et al. 2008). This metric captures the total amount of variation in trait values, making it conceptually analogous to PD $_{\text {Faith }}$ (Tucker et al. 2017). This metric is particularly commonly used in the ecological literature; alternative measures of trait diversity such as functional dendrograms (Petchey and Gaston 2006), or $\mathrm{ED}_{\mathrm{f}}$ (Faith 2015a) are also analogous richness metrics (Pavoine and Bonsall 2011) but have been less widely adopted. The convex hull based approach is widely used but its limitations should be noted: values calculated with different numbers of traits or species are not directly comparable, it is sensitive to outliers, and should not be used when the number of traits and species are similar (Podani 2009). We also calculated alternate metrics associated with the dimensions of divergence (traits: functional divergence, phylogeny: MPD) and regularity (traits: functional evenness, phylogeny: VPD) (Villéger et al. 2008, Tucker et al. 2017).

\section{Phylogenetic simulations}

We simulated phylogenetic trees of varying size (100 each with $16,32,64$, or 128 tips) using the sim.bdtree function from the R package geiger (Harmon et al. 2008) to generate 400 Yule trees. For each simulated tree, we used the mvMORPH R package (Clavel et al. 2015) to evolve sets of $1,2,4,6,8,10$, and 12 traits along the branches of the tree (Harmon et al. 2008). Here, we consider two common models of trait evolution (Appendix S1: Table S1).

1) Brownian Motion (BM). Sigma $\left(\sigma^{2}\right)$, the rate of trait divergence, was set to one of three different values: 0.2 , 0.75 , and 1.5. All traits were continuous, with an ancestral state of 0 , and simulated independently. We replicated each combination of tree and parameter values 100 times, producing a total of 8,400 unique trait-phylogeny sets.

2) Ornstein-Uhlenbeck (OU). Simulations used the same set of 400 Yule trees with matching sigma values, but this 
time we also varied alpha, the strength of selection in the OU model: alpha $=0.5,1.0$, and 2.0 , producing a total of 25,200 unique trait-phylogeny sets. This parameter space covers conditions for which FD might decline with trait number and increase with trait number (Appendix S2). We note that OU models have been shown to be essentially identical to models of accelerating rates of trait evolution.

\section{Null comparisons (star phylogenies)}

It is possible that the niche space occupied by a group of $n$ species is related to the amount of PD, but that this relationship simply reflects the number of species and their ages, rather than the specific ancestral relationships between the species in that group. We can estimate the degree to which the relationship between PD and FD might depend on this phylogenetic structure by comparison to null (star) phylogenies. The star phylogenies were constrained to have the same number of tips as those from the Yule trees, and the same total PD, but no internal phylogenetic structure. Traits were simulated for BM and OU models (parameter values shown in Appendix S1: Table S1). We simulated 30 replicates of each unique trait-tree combination (resulting in 2,520 traitphylogeny sets under BM and 7,560 under OU). Because all species are equidistant on the star trees, all species evolve independently, and these simulations therefore provide a null expectation for the relationship between PD and FD for a given tree size and model of evolution.

\section{Describing the relationship between $P D$ and FD}

FD values for each combination of tree size (i.e., number of taxa) and trait number were rescaled by their maximum FD value (final values between 0 and 1 ) to facilitate appropriate comparisons of the FD metric. We described the relationship between PD and FD in two ways. First, we calculated the Spearman rank correlation between the two variables, a commonly reported test statistic in the literature. Second, we more precisely describe the shape of the relationship between $\mathrm{PD}$ and FD by fitting non-linear least squares models to the simulated data (nls function in R), using the analytical expectations detailed in Appendix S2 to inform the shape of that relationship. For data generated across all BM simulations, an exponential growth model provided the best fit:

$$
\mathrm{FD} \sim a e^{1-b \mathrm{PD}}
$$

where $a$ reflects the intercept, and $b$ the slope or rate at which FD accumulates with increasing PD (See Kelly et al. [2014] for a similar approach). For data generated across all OU simulations, a logistic model provided the best fit:

$$
\mathrm{FD} \sim a+b \ln \mathrm{PD}
$$

where $b$ is the slope or rate at which FD accumulates with increasing PD, and $a$ is the intercept. We used these models to ask how well variability in FD is explained by PD, how the number of traits alters the shape of the relationship between PD and FD, and compare these to the null expectations found by fitting the same models to the data from the star trees.

\section{More complex models of trait evolution}

We additionally explored more complex models of trait evolution using the OUwie $\mathrm{R}$ package (Beaulieu and O'Meara 2014) to ask how the relationship between PD and FD is affected when different parts of a phylogeny experience different parameters of trait evolution. For the same set of phylogenetic trees described above, we identified two monophyletic partitions in the taxa: we first identified a bifurcation that roughly split the phylogeny into two similar sized clades, and varied the model of evolution between these sections. We then simulated scenarios in which (1) trait evolution occurs via BM, but sigma differs between two sections of the tree; (2) trait evolution occurs via OU but the optimal trait value (theta) varies between the two sections; (3) as in (2) but with both theta and sigma varying between the two sections; (4) trait evolution varies in terms of both theta and alpha between two sections; and (5) theta, alpha and sigma all vary between the two sections of the tree (see Appendix S1: Table S2 for parameter values used). Finally, we simulated an Early Burst (EB) model, on the same set of 400 Yule trees with matching sigma values as for the BM and OU simulations, but this time also varying beta, the exponential rate of decay: beta $=0.5,1.0$, and 2.0 , producing an additional total of 25,200 unique trait-phylogeny sets. Because parameter space rapidly becomes vast, we necessarily limited our exploration to ensure reasonable computing time.

\section{RESUlts}

Across the many simulated trees and traits, the relationship between PD and FD under BM was positive and monotonic, while under OU the relationship was positive and saturating. For both models of trait evolution, the relationship between PD and FD tended to increase as more traits were included in FD (Table 1, Fig. 1 insets). The null star trees had comparatively lower correlations between PD and FD (Table 1). We show here the results from the largest phylogenies (128 taxa); results were qualitatively similar for the smaller phylogenies (Appendix S1: Figs. S1-S6), but unsurprisingly became more variable as trees decreased in size.

We used non-linear least squares models to estimate the rate at which FD is accumulated (i.e., the slope of the relationship given by $b$ in Eq. 1 and 2), and measured the total variation $\left(R^{2}\right)$ explained by this relationship. As more traits were included, the slope parameter increased, suggesting the shape of the relationship between PD and FD depends on how many traits are being considered (Fig. 1a,b, main plots). For BM simulations, when only 1 or 2 traits were used to calculate FD, the relationship was close to linear, but became increasingly exponential with 4 or more traits (Fig. 1a, panels). For OU simulations the relationship between PD and FD changed from linear to saturating as more traits were included (Fig. 1b, panels). Finally, the total variation in FD explained by PD also depended strongly on the number of traits (Fig. 2). The $R^{2}$ improves rapidly with the addition of traits until approximately four traits. 
TABLE 1. Spearman's rank correlations between PD and FD, for each simulation scenario: BM and OU models of trait evolution, star tree (null) results, and multi-rate, multi-optima models (Scenarios 1-5, see Methods for details).

\begin{tabular}{|c|c|c|c|c|c|c|c|}
\hline Model & 1 trait & 2 traits & 4 traits & 6 traits & 8 traits & 10 traits & 12 traits \\
\hline $\mathrm{BM}$ & 0.355 & 0.528 & 0.711 & 0.736 & 0.767 & 0.730 & 0.700 \\
\hline $\mathrm{BM}$ star tree & -0.028 & 0.094 & -0.054 & 0.141 & 0.101 & -0.055 & 0.105 \\
\hline $\mathrm{OU}$ & 0.230 & 0.457 & 0.681 & 0.754 & 0.789 & 0.784 & 0.774 \\
\hline OU star tree & 0.063 & -0.032 & 0.070 & -0.063 & -0.075 & -0.088 & 0.0322 \\
\hline Scenario 1 & 0.340 & 0.501 & 0.680 & 0.753 & 0.776 & 0.876 & $\mathrm{n} / \mathrm{a}$ \\
\hline Scenario 2 & 0.211 & 0.299 & 0.370 & 0.389 & 0.435 & 0.461 & $\mathrm{n} / \mathrm{a}$ \\
\hline Scenario 3 & 0.235 & 0.278 & 0.341 & 0.381 & 0.416 & 0.438 & $\mathrm{n} / \mathrm{a}$ \\
\hline Scenario 4 & 0.249 & 0.351 & 0.422 & 0.469 & 0.489 & 0.496 & $\mathrm{n} / \mathrm{a}$ \\
\hline Scenario 5 & 0.246 & 0.325 & 0.399 & 0.443 & 0.469 & 0.477 & $\mathrm{n} / \mathrm{a}$ \\
\hline EB & 0.274 & 0.418 & 0.404 & 0.343 & 0.324 & 0.297 & 0.234 \\
\hline
\end{tabular}
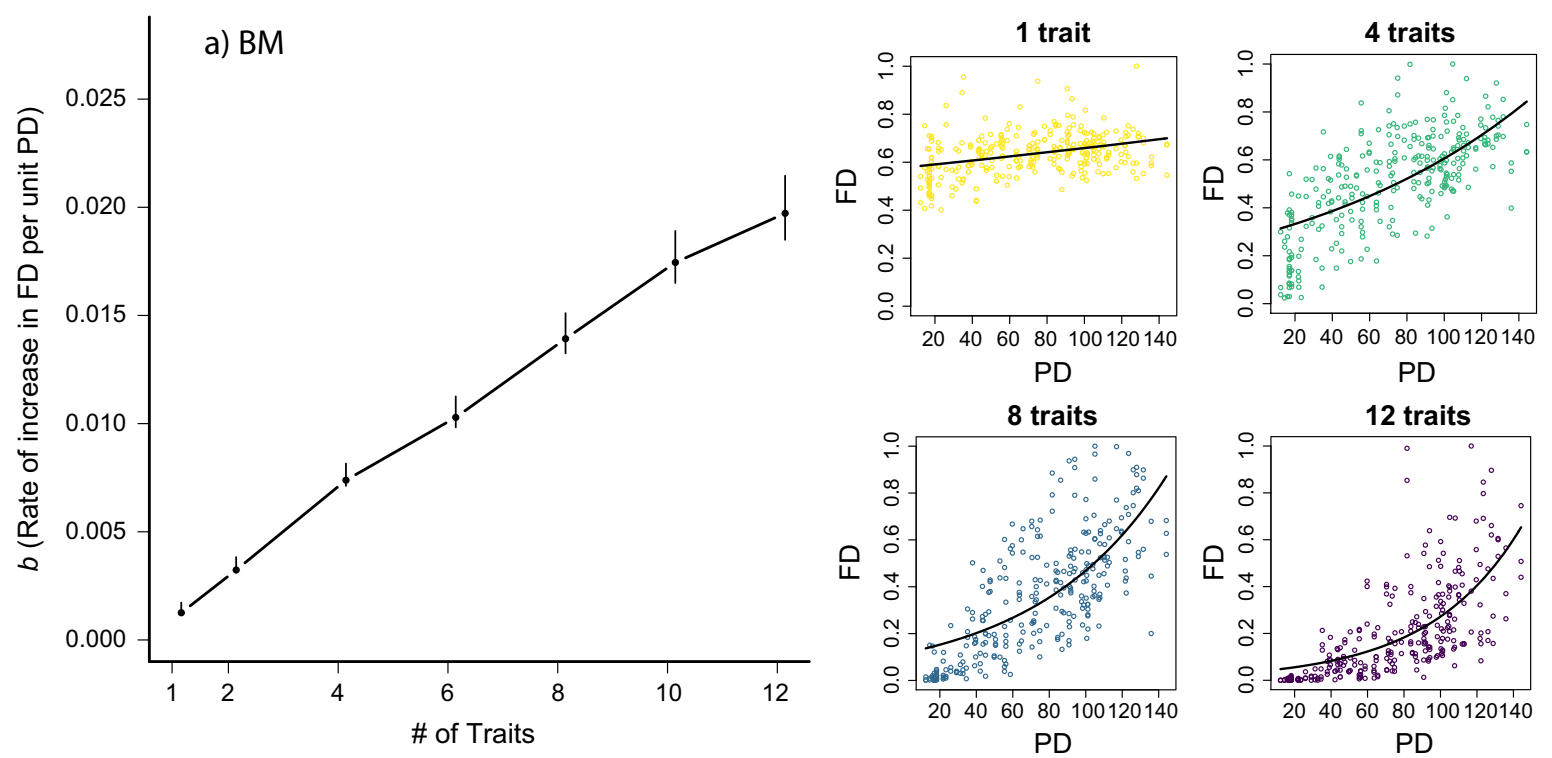

PD
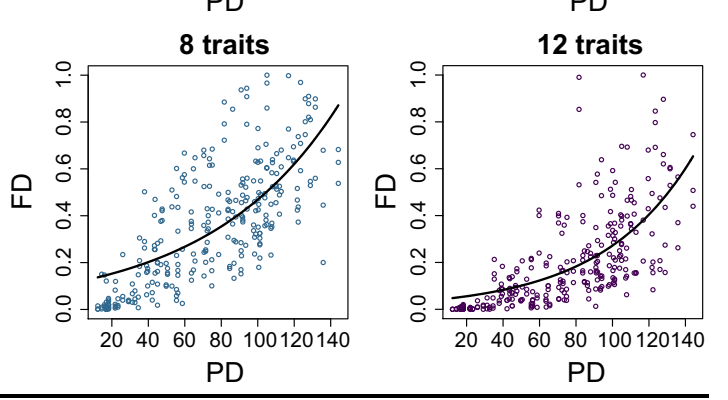

PD
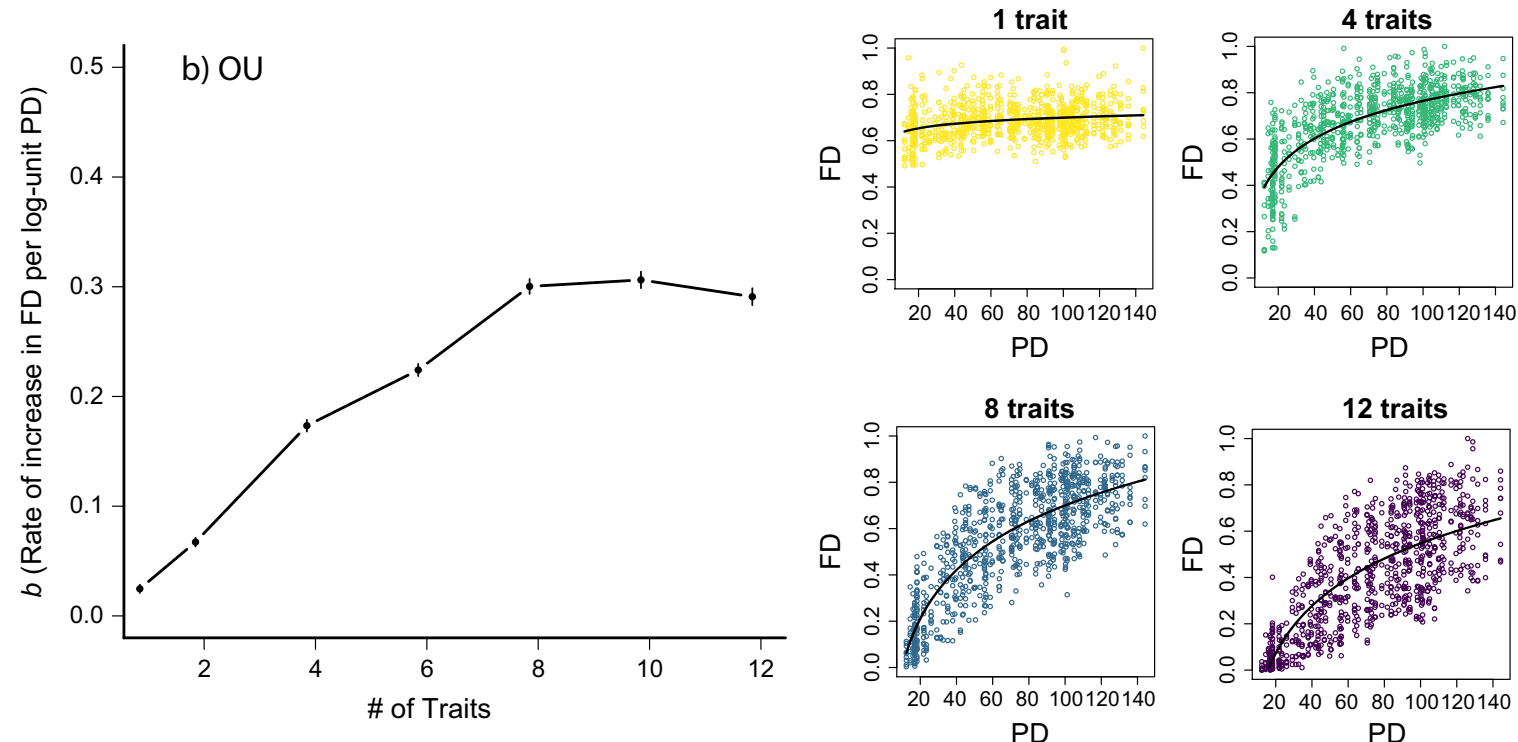

FIG. 1. The strength of the relationship between phylogenetic diversity (PD) and trait diversity (FD), across all simulations under the (a) Brownian motion and (b) Ornstein-Uhlenbeck models of trait evolution. In each panel, the main plot shows the estimated slope ( $b$ in Eqs. 1 and 2) by which FD increases per unit of PD (or $\log (\mathrm{PD})$ for $\mathrm{OU}$ ) in relationship to the number of traits. Surrounding plots show the raw data, and illustrate the change in the shape of the relationship between PD and FD for 1, 4, 8, or 12 traits. In each, note that FD is scaled relative to the maximum FD value, with scaling done separately for each combination of trait number, tree size, and model of evolution. 

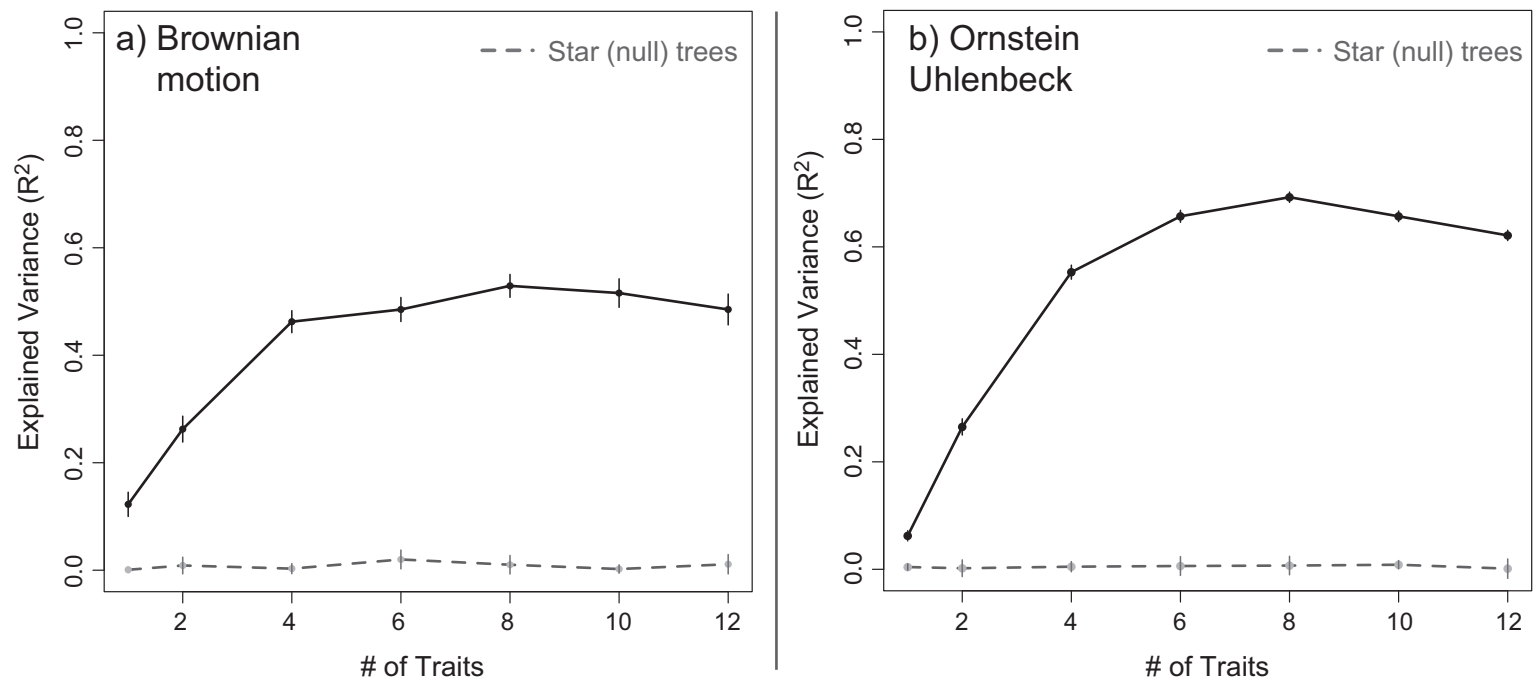

FIG. 2. The explained variance $\left(R^{2}\right)$ associated with the models described in Eqs. 1 and 2 for (a) Brownian motion and (b) Ornstein-Uhlenbeck, relating FD and PD for each number of traits. Solid lines show simulation result. Dashed lines show the comparable variance in FD explained by PD for a null scenario where individuals evolve independently along star trees with the same total PD. Error bars show standard deviations in $R^{2}$ values for 10,000 cross-validations in which $25 \%$ of the total data was omitted and the model refit to the remaining data.

However, PD always captures far greater variation in FD than the null expectation.

Outcomes from simulations of trait evolution with multiple rates or optima were generally similar in terms of the shape of the relationship between PD and FD to those seen under single-rate models (Appendix S1: Fig. S7) but weaker (Table 1), reflecting the greater variation in FD generated under these more complex models. The strength of the relationship $\left(R^{2}\right)$ between PD and FD calculated based on fitted Eqs. 1 and 2 was typically similar (BM) or weaker (OU) than that calculated for the single-rate simulations (Appendix S1: Fig. S7). The slope of the relationship between PD and FD also generally increased with trait number, though this relationship was weaker for the multi-optima/multi-rate $\mathrm{OU}$ models compared to the single-rate OU. Results from the EB simulations were the exception, for which the relationship between PD and FD was neither strong nor monotonic (see Table 1 and Appendix S1: Fig. S8).

\section{Discussion}

Phylogenetic diversity has frequently been used as a surrogate for trait diversity in ecological analyses. Surprisingly, however, the expected relationship between multi-dimensional trait diversity and phylogenetic diversity has not been well described, despite the common practice of using multiple traits to estimate FD. Here, we used numerical simulations to explore how trait dimensionality alters the relationship between PD and FD, assuming either a Brownian motion or Ornstein-Uhlenbeck model of evolution. We show that the strength and shape of the PD-FD relationship is tied to trait dimensionality. PD correlates relatively poorly with FD when estimated using only a single trait, although a single trait still performed better than our null expectation (star trees). This result is realized on a large phylogeny (128 taxa) with many replicates, but the relationship between PD and FD will be weaker and more variable for smaller phylogenies (e.g., Appendix S1: Fig. S5). It is not surprising that studies using PD as a proxy for single traits are therefore liable to find weak or null results, irrespective of the true underlying process (Letten and Cornwell 2015, Cadotte et al. 2017). For example, studies on character displacement may find misleading results since they frequently focus on single traits (Dayan and Simberloff 2005), and phylogenetic diversity is likely a poor surrogate for variation in a single trait (and a single trait likely a poor estimator of niche occupancy). However, with increasing numbers of traits, PD correlates increasingly strongly and positively with FD. In cases where many traits affect ecological processes, such as species' coexistence (Kraft et al. 2015), PD is a potentially useful tool with which to estimate trait diversity, particularly in the absence of trait data. This does not mean that PD is an effective surrogate for missing traits (although it might be), but rather that it is effective in the absence of trait data at estimating multidimensional differences between species, at least for commonly assumed models of evolution.

This surrogacy value of phylogenetic diversity arises when the phylogenetic structure captures an assemblage's evolutionary history. In contrast, although the null phylogenies contained the same total amount of evolutionary history, they provided no information about the relative trait differences among species and so provided no information on trait diversity. The relative proportions of shared and unshared branch lengths on a phylogeny contribute importantly to the expected trait diversity of a group of species. This was true for the common single rate models of trait evolution we considered, BM and OU. Comparisons of evolutionary models fit to observed trait values most often find support for $\mathrm{OU}$ and BM, with support for models such as 'early burst' (EB) being much less common (Harmon et al. 2010, Pennell et al. 2015). Thus, at least for these simple models (see below for discussion of more complex scenarios), it is reasonable to assume that PD and multidimensional measures of FD should represent similar information about the extent of relative differences among a set of taxa. 
While our results imply that the relationship between PD and FD can be a useful tool for ecologists, recent papers have highlighted the varying strength of observed relationships between measures of trait diversity and phylogenetic diversity (see discussion in Cadotte et al. 2017). A strong relationship may not be the case when increasingly complex models of trait evolution (Appendix S1: Fig. S7) are relevant, although multi-rate BM maintained a strong correlation between PD and FD. Kelly et al. (2014) found a weak relationship between $\mathrm{PD}$ and $\mathrm{FD}$, in part because they included a large number of traits with discrete character states for which (unlike the continuous data we examine here) saturation in state changes tend to quickly mask the PD-FD relationship. Others have argued that the combination of traits can cause the masking of phylogenetic structure; our results support this intuition (Murrell 2017). In addition, as species pools are sorted into local communities, the relationship between PD and FD may change greatly. For example, even in the absence of ecological processes, subsets of a clade can have a much different relationship between PD and FD than that observed at the clade scale (Mazel et al. 2017). Here we attempt to establish a baseline expectation for the strength and shape of the PD-FD relationship at the clade-level, against which observed relationships can be appropriately compared.

A higher amount of trait diversity is often assumed to indicate greater separation in niche space, as long as differences in trait values correspond to different positions along a niche axis (Violle and Jiang 2009, Garnier et al. 2016). When this assumption holds, FD and PD should provide related information regarding occupancy of niche space. This is most likely when traits contribute equally to ecological differences and trait values directly relate to niche axes. However, ecologically relevant traits are frequently complex. They may represent the products of suites of co-evolving traits (e.g., leaf mass per unit area) and measures of FD may include traits with differing complexities. Some traits more directly relate to niche axes than others - for example, measures of height or root depth in plants are frequently linked to niche differentiation or limiting similarity to minimize competitive interactions (Garnier et al. 2016). Other traits, such as nutrient ratios in plant tissues, may be less directly related to niche axes. However, in concert with other traits as a result of tradeoffs and constraints, they can contribute to life history axes that are related to niche differences (Wright et al. 2004, Reich 2014, Díaz et al. 2016). Other traits may contribute little-directly or indirectly - to ecological differences (Shipley et al. 2016) — in which case both FD and PD will be poor estimates of niche space. Finally, ecological responses of interest such as competitive intensity (Mayfield and Levine 2010) or ecological complementarity (Cardinale et al. 2012) may be poorly captured by measures of trait diversity.

Although categorizing relevant niche axes remains elusive, recent work indicates that multiple traits are necessary to describe a species' ecological niche (Laughlin 2014), and at a global scale, Díaz et al. (2016) found that two axes representing six traits were needed to capture the major variation in structure and function across plant groups. It is reassuring that concordance between measures of PD and FD increases when calculated at with multiple traits. However, further work formally connecting traits and the niche concept, and considering the connections between these and the environment remains necessary. The relationship between niche space and other dimensions of trait and phylogenetic diversity are even less clear, and supplementary results suggest that when considering measures of evenness or divergence, trait and phylogenetic facets may not correspond (Appendix S1: Figs. S9-S10) due to sensitivity of these dimensions to phylogenetic topology and the model of trait evolution. The key challenge for the future then is to better understand how ecologically relevant traits match to the assumed models of evolution, and how ecological processes map onto both PD and FD.

\section{ACKNOWLEDGMENTS}

We thank the Canadian Institute for Ecology and Evolution for funding the Synthesizing Conservation and Phylogenetics workshop that inspired this paper. We thank all workshop members, particularly Arne Mooers, Matt Pennell, and Florent Mazel, for discussions related to this paper. We thank Dan Faith and 2 anonymous reviewers for their comments and advice. CMT acknowledges the European Commission for the Marie Curie IIF (H2020-MSCA-IF2014-657951). MWC was supported by the TD Professor of Urban Forest Conservation and Biology chair, Canada Foundation for Innovation, the Ontario Research Fund, and Natural Sciences and Engineering Research Council of Canada (\#386151).

\section{Literature Cited}

Ackerly, D. 2009. Conservatism and diversification of plant functional traits: evolutionary rates versus phylogenetic signal. Proceedings of the National Academy of Sciences of the United States of America 106:19699-19706.

Beaulieu, J., and B. O’Meara. 2014. OUwie: analysis of evolutionary rates in an OU framework. $\mathrm{R}$ package version 1.

Blonder, B., C. Lamanna, C. Violle, and B. J. Enquist. 2014. The n-dimensional hypervolume. Global Ecology and Biogeography 23:595-609.

Cadotte, M. W., B. J. Cardinale, and T. H. Oakley. 2008. Evolutionary history and the effect of biodiversity on plant productivity. Proceedings of the National Academy of Sciences of the United States of America 105:17012-17017.

Cadotte, M. W., J. Cavender-Bares, D. Tilman, and T. H. Oakley. 2009. Using phylogenetic, functional and trait diversity to understand patterns of plant community productivity. PLoS ONE 4:e5695.

Cadotte, M. W., J. D. Davies, and P. R. Peres-Neto. 2017. Why phylogenies do not always predict ecological difference. Ecological Monographs 87:535-551.

Cardinale, B. J., J. E. Duffy, A. Gonzalez, D. U. Hooper, C. Perrings, P. Venail, A. Narwani, G. M. Mace, D. Tilman, and D. A. Wardle. 2012. Biodiversity loss and its impact on humanity. Nature 486:59-67.

Chase, J. M., and M. A. Leibold. 2003. Ecological niches: linking classical and contemporary approaches. University of Chicago Press, Chicago, Illinois, USA.

Chesson, P. 2000. Mechanisms of maintenance of species diversity. Annual Review of Ecology and Systematics 31:343-366.

Clavel, J., G. Escarguel, and G. Merceron. 2015. mvMORPH: an R package for fitting multivariate evolutionary models to morphometric data. Methods in Ecology and Evolution 6:1311-1319.

Cornwell, W. K., D. W. Schwilk, and D. D. Ackerly. 2006. A TRAIT-BASED TEST FOR HABITAT FILTERING: CONVEX HULL VOLUME. Ecology 87:1465-1471.

Davies, T. J., and L. B. Buckley. 2011. Phylogenetic diversity as a window into the evolutionary and biogeographic histories of present-day richness gradients for mammals. Philosophical Transactions of the Royal Society B: Biological Sciences 366:24142425 . 
Dayan, T., and D. Simberloff. 2005. Ecological and communitywide character displacement: the next generation. Ecology Letters 8:875-894.

Devictor, V., D. Mouillot, C. Meynard, F. Jiguet, W. Thuiller, and N. Mouquet. 2010. Spatial mismatch and congruence between taxonomic, phylogenetic and functional diversity: the need for integrative conservation strategies in a changing world. Ecology Letters 13:1030-1040.

Díaz, S., et al. 2016. The global spectrum of plant form and function. Nature 529:167-171.

Elton, C. 1927. Animal ecology. Sidgwick and Jackson, London, UK.

Faith, D. P. 1992. Conservation evaluation and phylogenetic diversity. Biological Conservation 61:1-10.

Faith, D. P. 2015a. Phylogenetic diversity, functional trait diversity and extinction: avoiding tipping points and worst-case losses Philosophical Transactions of the Royal Society of London. Series B, Biological Sciences 370:20140011.

Faith, D. P. 2015b. The unimodal relationship between species' functional traits and habitat gradients provides a family of indices supporting the conservation of functional trait diversity. Plant Ecology 216:725-740.

Faith, D. P. 2016. The PD phylogenetic diversity framework: linking evolutionary history to feature diversity for biodiversity conservation. Pages 39-56 in R. Pellens, and P. Grandcolas, editors. Biodiversity conservation and phylogenetic systematics. Springer, Sorbonne, France.

Flynn, D. F., N. Mirotchnick, M. Jain, M. I. Palmer, and S. Naeem. 2011. Functional and phylogenetic diversity as predictors of biodiversity-ecosystem-function relationships. Ecology 92:15731581 .

Futuyma, D. J. 2010. Evolutionary constraint and ecological consequences. Evolution 64:1865-1884.

Garnier, E., M.-L. Navas, and K. Grigulis. 2016. Plant functional diversity. Oxford University Press, Oxford, UK.

Gerhold, P., J. F. Cahill, M. Winter, I. V. Bartish, and A. Prinzing. 2015. Phylogenetic patterns are not proxies of community assembly mechanisms (they are far better). Functional Ecology 29:600614.

Grinnell, J. 1924. Geography and evolution. Ecology 5:225-229.

Harmon, L. J., J. T. Weir, C. Brock, R. E. Glor, and W. Challenger. 2008. GEIGER: investigating evolutionary radiations. Bioinformatics 24:129-131

Harmon, L. J., J. B. Losos, T. Jonathan Davies, R. G. Gillespie, J. L. Gittleman, W. Bryan Jennings, K. H. Kozak, M. A. McPeek, F. Moreno-Roark, and T. J. Near. 2010. Early bursts of body size and shape evolution are rare in comparative data. Evolution 64:2385-2396.

Helmus, M. R., W. Keller, M. J. Paterson, N. D. Yan, C. H. Cannon, and J. A. Rusak. 2010. Communities contain closely related species during ecosystem disturbance. Ecology Letters 13:162-174.

Hutchinson, G. E. 1961. The paradox of the plankton. American Naturalist 95:137-145.

Kelly, S., R. Grenyer, and R. W. Scotland. 2014. Phylogenetic trees do not reliably predict feature diversity. Diversity and Distributions 20:600-612.

Kembel, S. W., and S. P. Hubbell. 2006. The phylogenetic structure of a neotropical forest tree community. Ecology 87:S86-S99.

Kraft, N. J. B., W. Cornwell, C. O. Webb, and D. D. Ackerly. 2007. Trait evolution, community assembly, and the phylogenetic structure of ecological communities. American Naturalist 170:271283.

Kraft, N. J., O. Godoy, and J. M. Levine. 2015. Plant functional traits and the multidimensional nature of species coexistence.
Proceedings of the National Academy of Sciences of the United States of America 112:797-802.

Laughlin, D. C. 2014. The intrinsic dimensionality of plant traits and its relevance to community assembly. Journal of Ecology 102:186-193.

Letten, A. D., and W. K. Cornwell. 2015. Trees, branches and (square) roots: why evolutionary relatedness is not linearly related to functional distance. Methods in Ecology and Evolution 6:439-444.

Mayfield, M. M., and J. M. Levine. 2010. Opposing effects of competitive exclusion on the phylogenetic structure of communities. Ecology Letters 13:1085-1093.

Mazel, F., A. O. Mooers, G. V. Dalla Riva, and M. W. Pennell. 2017. Conserving phylogenetic diversity can be a poor strategy for conserving functional diversity. Systematic Biology 66:1019-1027.

McGill, B., B. J. Enquist, E. Weiher, and M. Westoby. 2006. Rebuilding community ecology from functional traits. Trends in Ecology and Evolution 21:178-186.

Meynard, C. N., V. Devictor, D. Mouillot, W. Thuiller, F. Jiguet, and N. Mouquet. 2011. Beyond taxonomic diversity patterns: how do $\alpha, \beta$ and $\gamma$ components of bird functional and phylogenetic diversity respond to environmental gradients across France? Global Ecology and Biogeography 20:893-903.

Murrell, D. J. 2017. A global envelope test to detect early and late bursts of trait evolution. bioRxiv 175968 .

Pavoine, S., and M. Bonsall. 2011. Measuring biodiversity to explain community assembly: a unified approach. Biological Reviews 86:792-812.

Pennell, M. W., R. G. FitzJohn, W. Cornwell, and L. J. Harmon. 2015. Model adequacy and the macroevolution of angiosperm functional traits. American Naturalist 186:E33-E50.

Petchey, O. L., and K. J. Gaston. 2006. Functional diversity: back to basics and looking forward. Ecology Letters 9:741-758.

Podani, J. 2009. Convex hulls, habitat filtering, and functional diversity: mathematical elegance versus ecological interpretability. Community Ecology 10:244-250.

Reich, P. B. 2014. The world-wide 'fast-slow'plant economics spectrum: a traits manifesto. Journal of Ecology 102:275-301.

Shipley, B., F. De Bello, J. H. C. Cornelissen, E. Laliberté, D. C. Laughlin, and P. B. Reich. 2016. Reinforcing loose foundation stones in trait-based plant ecology. Oecologia 180:923-931.

Silvertown, J., K. McConway, D. Gowing, M. E. Dodd, M. F. Fay, J. A. Joseph, and K. Dolphin. 2006. Absence of phylogenetic signal in the niche structure of meadow plant communities. Proceedings of the Royal Society of London. Series B 273:39-44.

Srivastava, D. S., M. W. Cadotte, A. A. M. MacDonald, R. G. Marushia, and N. Mirotchnick. 2012. Phylogenetic diversity and the functioning of ecosystems. Ecology Letters 15:637-648.

Tucker, C. M., et al. 2017. A guide to phylogenetic metrics for conservation, community ecology and macroecology. Biological Reviews 92:698-715.

Villéger, S., N. W. Mason, and D. Mouillot. 2008. New multidimensional functional diversity indices for a multifaceted framework in functional ecology. Ecology 89:2290-2301.

Violle, C., and L. Jiang. 2009. Towards a trait-based quantification of species niche. Journal of Plant Ecology 2:87-93.

Violle, C., M. L. Navas, D. Vile, E. Kazakou, C. Fortunel, I. Hummel, and E. Garnier. 2007. Let the concept of trait be functional!. Oikos 116:882-892.

Webb, C. O., D. D. Ackerly, M. A. McPeek, and M. J. Donoghue. 2002. Phylogenies and community ecology. Annual Review of Ecology, Evolution, and Systematics 33:475-505.

Wright, I. J., et al. 2004. The worldwide leaf economics spectrum. Nature 428:821-828.

\section{SUPPORTING INFORMATION}

Additional supporting information may be found in the online version of this article at http://onlinelibrary.wiley.com/doi/10.1002/ecy. 2349/suppinfo 